\title{
Thermal Cycling and Growth of the Martensite Studied by NMR in an Ag-Cd Alloy
}

\author{
P. Scherrer, S. Rubini*, C. Dimitropoulos and F. Borsa* \\ Institut de Physique Expérimentale, EPFL, CH-1015 Lausanne, Switzerland \\ * Dipartimento di Fisica "A. Volta", Universita di Pavia, I-27100 Pavia, Italy
}

\begin{abstract}
Cd}$ NMR measurements in two Ag-Cd samples cycled between room temperature and temperatures below $M_{s}$ are presented. The growth curve of the martensite on cooling presents a discontinuity in the cycling temperature region.

It is shown that the method of analysis of the NMR data recently developed by us [Phys.Rev.B 46, 10563 (1992)], allows to detect subtle memory effects and the absolute determination of the fraction of the martensite in thermoelastic equilibrium with the parent phase at fixed temperature.
\end{abstract}

\section{INTRODUCTION}

Nuclear Magnetic Resonance (NMR) has proved to be a powerful method to monitor the evolution of Martensitic Phase Transformations (MPT) in shape memory alloys ${ }^{1}$. Studies of NMR absorption-spectra not only yield interesting information about the local electronic and cristallographic structure but also allow to determine the exact transformation path of the martensitic phase.

Recently, micromemory effects in shape memory alloys, following incomplete thermal cycling on heating (i.e. on reverse transformation), have been reported ${ }^{2}$. Similar effects have now been evidenced in an AgCd alloy upon incomplete cycling on cooling (ICC). The transformation path between the austenitic phase and the martensitic phase has been deduced from ${ }^{113} \mathrm{Cd}$ NMR absorption-spectra. Attention was focused on the effects of ICC on the martensite growth curve.

\section{EXPERIMENTAL PROCEDURE}

The alloy used in the present experiment, an Ag-46.5at\% Cd was prepared by melting at about $1270 \mathrm{~K}$ known weights of $\mathrm{Ag}$ (99.9995 pct) and Cd (99.9998 pct) in a sealed quartz tube filled with argon. The molten alloy was well mixed by vigorously agitating the melt. It was then quickly cooled to room temperature to minimize segregation. The loss of weight after melting was less than $0.07 \%$ and so chemical analysis was not made. After removing the oxyde surface, the ingot was homogenized at $953 \mathrm{~K}$ in vacuum and subsequently quenched into iced water to retain the $\beta$-phase. The mean grain size of the polycrystalline samples is approximately $10 \mu \mathrm{m}$.

Differential Scanning Calorimetry (DSC) was used to measure the stabilized transition temperatures. They have been determined to be $M_{s}=143 \mathrm{~K}$ and $\mathrm{M}_{\mathrm{f}} \approx 123 \mathrm{~K}$ as aiready reported in earlier works 3,4 .

Samples for NMR measurements were prepared by cutting the ingot into small cubes $(0.3 \mathrm{~mm}$ per side) with a multiple diamond-wire saw and mixing these cubes with powdered quartz. Two ICC stop temperatures have been selected at $T_{S 1}=78 \mathrm{~K}$ and $T_{S 2}=131 \mathrm{~K}$. Thermal cycling was performed in a first step by quenching the sample 20 times into liquid nitrogen and heating it back to room temperature. After measurement, in a second step, the same sample was cycled 20 times from room temperature to $T_{S 2}$ by quenching it into a cryogenic bath of methylcyclopentane in equilibrium at its fusion temperature. One sample was used as reference and therefore not cycled. 
Standard pulse-NMR techniques were applied for ${ }^{113} \mathrm{Cd}$ spin-echo acquisition, the measurements being made in equilibrium at a fixed temperature and with a static magnetic field $\mathrm{H}_{0}=8 \mathrm{~T}$. Fourier Transform of the decreasing half of the echo leads to the NMR absorption spectrum.

\section{RESULTS AND DISCUSSION}

Studies of the ${ }^{113} \mathrm{Cd}$ NMR absorption-spectrum 5 as function of temperature have shown that the absorption line transforms from a single Gaussian line above $M_{s}$ (see Fig. 1a) to a doublet during the martensitic phase transformation. The new line, corresponding to the growing martensite, arises at a higher frequency than that of the original line which corresponds to the austenite. During transformation, the amplitude of this martensite-related line increases while the line corresponding to the austenite decreases (see Fig. 1b, c). Therefore a visual monitoring of the martensitic phase growth is possible although the volumic fraction of martensite present at a given temperature may not be directly deduced from the spectrum itself. At very low temperature $(T<30 \mathrm{~K})$, on completion of the MPT, only one Gaussian line representing the martensitic phase remains on the absorption-spectrum (see Fig. 1d).
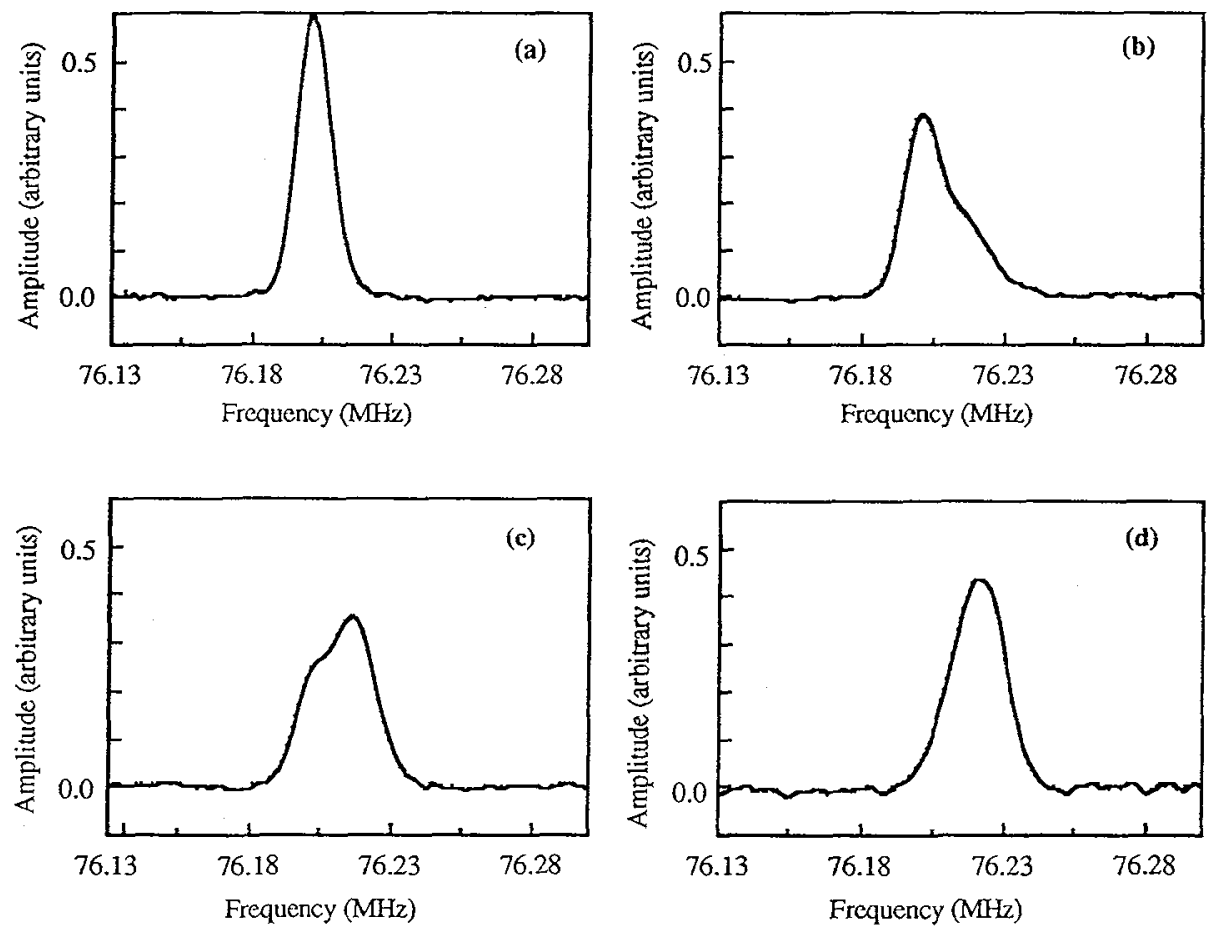

Fig. 1: ${ }^{113} \mathrm{Cd} N \mathrm{MR}$ absorption-spectra in Ag-46.5at\% Cd, $\mathrm{H}_{\mathrm{O}}=8 \mathrm{~T}$ (a) $\mathrm{T}=156 \mathrm{~K}\left(>\mathrm{M}_{\mathrm{S}}\right)$, (b) $\mathrm{T}=141.5 \mathrm{~K}\left(<\mathrm{M}_{\mathrm{S}}\right)$, (c) $\mathrm{T}=133 \mathrm{~K}$, (d) $\mathrm{T}=30 \mathrm{~K}$

To determine the fraction of martensite as function of temperature, a deconvolution of the ${ }^{113} \mathrm{Cd}$ spectra is performed. The spectrum at a fixed temperature is separated into its two components by a fit of two Gaussian lines 5,6 , clearly pointing out the contribution of each one of the two coexisting phases to the global signal (see Fig. 2). The volumic fraction of martensite is calculated by comparing the product amplitude $\mathrm{x}$ width at half height of the two spectral lines.

The growth curves of martensite for the three studied cases are reported in Fig. 3. The reference sample shows an explosive nucleation at $M_{s}$, where about $40 \%$ of the austenitic phase transforms to martensite within a temperature drop of only $2 \mathrm{~K}$. The transformation subsequent to this explosive growth proceeds with a decreasing rate up to a fraction of $80 \%$ of martensite at $\mathrm{M}_{\mathrm{f}}$, as determined by DSC, and 
$95 \%$ at $40 \mathrm{~K}$. This behaviour reminds the characteristics of a burst kinetic mode ${ }^{7}$ in which the burst is followed by an athermal transformation.

The first ICC stop temperature, $T_{S 1}$, is situated in a zone where the transformation is normally almost achieved ( $90 \%$ of martensite). Nevertheless, the transformation path of the sample cycled to $\mathrm{T}_{\mathrm{S} 1}$ displays well visible traces of the thermal cycling. The fraction transformed by burst is reduced to about $30 \%$ but the immediatly subsequent growth is very similar to the reference path, maintaining almost constantly the initial fraction difference of $10 \%$. From $100 \mathrm{~K}$ down to $T_{S 1}$, expansion of the martensitic phase is very slow and the fraction is roughly constant. By overcooling to lower temperatures the growth resumes and the reference transformation path is catched up with. This transformation step being located below $120 \mathrm{~K}$, it is not detectable by DSC. These characteristics are retained by the transformation path of the same sample after ICC down to $\mathrm{T}_{\mathrm{S} 2}$ has been performed.

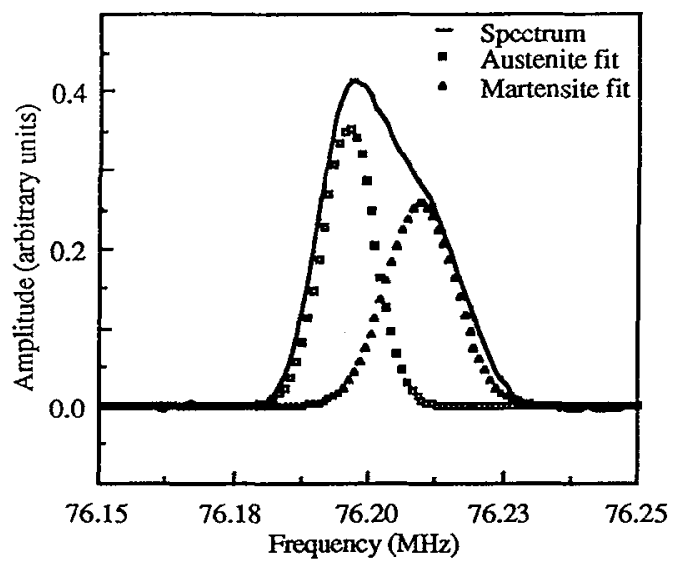

Fig.2: Deconvolution of the ${ }^{113} \mathrm{Cd}$ absorption spectrum $(\mathrm{T}=139 \mathrm{~K})$

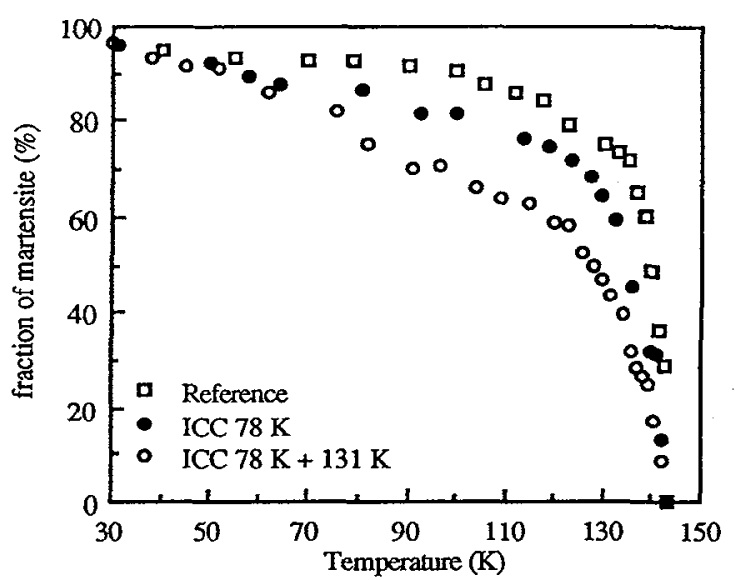

Fig.3: Transformed fraction of Martensite on cooling

As a consequence of the second cycling the fraction of martensite produced by explosive nucleation is further reduced and the fraction difference with respect to the preceeding path is maintained between $\mathrm{T}_{\mathbf{S} 2}$ and $T_{S 1}$. For temperatures slightly higher than $T_{S 2}$, the expected step in the growth path can be easily seen on the magnification of that curve portion (see Fig. 4a). Confirmation of the existence of this step is given by DSC measurements now clearly bearing a local minimum at the temperature corresponding to the beginning of the step (see Fig. $4 b, c)$.
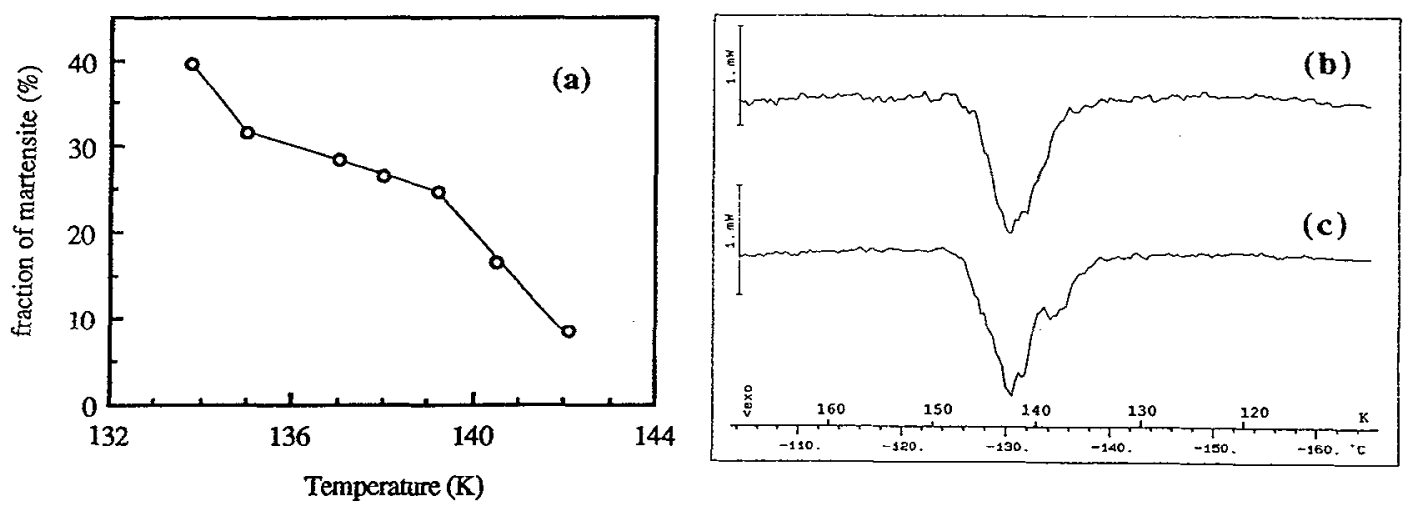

Fig. 4: Step on the transformation path as consequence of ICC to TS2 revealed by NMR (a) and by DSC before (b) and after cycling (c). 
It is to note that all these "fingerprints" of ICC are not reversible on performing the complete transformation several times.

\section{CONCLUSION}

The difference of ${ }^{113} \mathrm{Cd}$ NMR resonance frequency in the two coexisting phases, during the martensitic phase transformation in Ag-Cd alloy, allows an accurate determination of the martensite growth curve. Characteristical features of the transformation path are an initially burstlike martensite nucleation, followed by a slower growth up to almost fully transformed martensite.

Subtle memory effects have been detected upon incomplete cycling on cooling (forward transformation). The growth path is therefore bearing traces of the samples history. The main characteristics of these ICC "fingerprints" may be summarized as:

(a) the fraction of martensite transformed by explosive nucleation is reduced and this initial fraction difference with respect to the reference is maintained down to ICC stop temperature.

(b) the transformation proceeds stepwise above the ICC stop temperature

(c) these micromemory effects are not reversible

Further investigations are being carried out actually to connect the observed effects to transformation kinetics theories.

\section{ACKNOWLEDGMENT}

Financial assistance provided by the Fonds national suisse de la recherche scientifique of Switzerland is gratefully acknowledged.

\section{REFERENCES}

[1] Rubini S., Dimitropoulos C. and Borsa F., "Study of the Martensitic Transformation in a Ag-Cd alloy by "111 Cd NMR", 26. Congress Ampere (edited by Anagnostopoulos A., Milia F. and Simonopoulos A. , Athens, 1990)

[2] Airoldi G., Besseghini S. and Riva G., Il Nuovo Cimento $15 D$ (1993) 365

[3] Masson D.B., Trans. Metall. Soc. of AIME 218 (1960) 94

[4] Krishnan R.V. and Brown L. C., Metall. Trans. 4 (1973) 1017

[5] Rubini S., Dimitropoulos C. and Borsa F., Phys. Rev. B 49 (1994) 12590

[6] Rubini S., PhD Thesis, EPF-Lausanne, Switzerland (1992)

[7] Raghavan V., "Kinetics of Martensitic Transformations" in Martensite, Edited by Olson G.B. and Owen W.S. (ASM International 1992) 197 